\title{
Screening of hydrogen bond acceptor for the synthesis of low transition temperature mixtures with malic acid
}

\author{
C. L. Yiin ${ }^{1}$, S. Yusup ${ }^{1}$, A. T. Quitain ${ }^{2} \&$ Y. Uemura ${ }^{1}$ \\ ${ }^{1}$ Center of Biomass and Biofuels Research, \\ Universiti Teknologi PETRONAS, Malaysia \\ ${ }^{2}$ Graduate School of Science and Technology, Kumamoto University, \\ Japan
}

\begin{abstract}
Biomass exploitation emerges to be the most probable and practical alternative in solving the global depletion of fossil fuels. Nevertheless, the components in lignocellulosic biomass are assembled in a complex three-dimensional structure which leads to difficulty in hydrolyzation and resistance against chemicals. In this context, low transition temperature mixtures (LTTMs) tend to be the promising green solvents to delignify lignocellulosic biomass. This research focused on customizing the physicochemical behaviour of LTTMs by a prudent selection of hydrogen bond acceptor (HBA) in which malic acid is used as the hydrogen bond donor (HBD). LTTMs with carbohydrates as HBA could be formed in the temperature range of 60 to $100^{\circ} \mathrm{C}$. On the other hand, LTTMs with organic and inorganic salts as HBA could only be formed in the temperature range of 100 to $180^{\circ} \mathrm{C}$. Besides, no crystallization of starting materials was observed for LTTMs prepared from carbohydrates when they are cooled to room temperature. This phenomenon was due to the hydrogen bonding and van der Waals interactions formed between the starting materials. Based on Fourier transform infrared spectroscopy (FTIR) analysis, the representative peak of carboxylic acid group of malic acid turned broader. The peaks involved in the $\mathrm{H}$-bonding as a result of the formation of LTTMs shifted and became broader by the combination of malic acid and carbohydrates.
\end{abstract}

Keywords: hydrogen bond acceptor, hydrogen bond donor, low transition temperature mixtures (LTTMs), Fourier transform infrared spectroscopy, hydrogen bond. 


\section{Introduction}

Biomass utilization stands out as having the most potential and feasibility in harnessing its energy. Nilsen et al. [1] highlighted that unlike other renewable sources of energy such as solar and wind which only generates heat and power, biomass can be converted to liquid, solid and gaseous fuels. The components in lignocellulosic biomass such as lignin, cellulose and hemicellulose are assembled in a complex three-dimensional structure which lead to resistant against chemicals and microbial attack that makes it very difficult to hydrolyze. An effective dissociation of these components and their subsequent separation are needed for the production of high value products from lignocellulosic biomass [2].

Traditionally, strong base or mineral acids are used to exploit the lignocellulosic materials. The extracted fractions from these processes are severely and irreversibly altered and environmental pollution is also of great concern. Besides, conventional processes cannot fully utilize the non-cellulose biopolymer such as lignin and hemicelluloses. Hence, mild processes and pretreatment are proposed in order to overcome the aforementioned matters [3, 4]. However, this issue is still pending due to high crystallinity of cellulose, relatively high reactivity of carbohydrates, insolubility of cellulose in conventional solvents and heterogeneity of lignocelluloses [5]. Current processes to convert lignocellulosic into useful raw materials are highly inefficient. Even the most efficient processes, the production of paper from wood, show a biomass utilization of only 35\% [6]. In the future, bio-economy, finding a suitable solvent for lignocellulosic biomass is becoming crucial for biofuels processing. Hence, new technologies should be developed for the green bio-refinery of biomass resources.

Conventional methods used to dissociate biomass into cellulose, hemicelluloses and lignin bio-products often require extreme and expensive techniques. Although some ionic liquids were found to succeed as solvents using milder conditions, ionic liquids for large scale application still show limitations in terms of recoverability and cost [7]. Despite their high efficiency in dissolution of cellulose, new green approaches towards the replacement of volatile organic solvents were taken into consideration. A new family of solvents which can be known as "deep eutectic solvents (DES)" was introduced by Abbott et al. [8] as suitable alternative for ionic liquids. However, this name does not cover the classes of solvents because many of them show glass transition points instead of eutectic melting points which are also known as "low transition temperature mixtures (LTTMs)” [9]. These new solvents can be formed by mixing two solid starting materials which form a liquid through hydrogen bond interactions. The principles are not easy to generalize. The proton affinity (PA)/pKa equalization plays a role in strengthening the $\mathrm{H}$-bond, so the pKa slide rule was taken into account in the selection of the H-bonding counterparts [10].

Therefore, this research focused on customizing the physicochemical behaviour of LTTMs by a prudent selection of hydrogen bond acceptor (HBA) in which malic acid was used as the hydrogen bond donor (HBD). Experiments were conducted to investigate the compatibility of organic salts (ammonium acetate, sodium acetate), inorganic salt (ammonium sulfate) and carbohydrates (glucose, 
fructose, sucrose) as HBA. In addition, Fourier transform infrared spectroscopy (FTIR) analysis was conducted to ensure the existence of hydrogen bond in the synthesized LTTMs.

\section{Materials and methods}

\subsection{Materials}

The hydrogen bond donor (HBD) and hydrogen bond acceptor (HBA) which have been used as starting materials for the synthesis of LTTMs are listed in Table 1.

Table 1: Starting materials for the synthesis of LTTMs.

\begin{tabular}{|c|c|c|c|}
\hline \multirow{2}{*}{$\begin{array}{c}\text { Hydrogen bond } \\
\text { donor (HBD) }\end{array}$} & \multicolumn{3}{|c|}{ Hydrogen bond acceptor (HBA) } \\
\cline { 2 - 4 } & Inorganic acid & Organic acid & Carbohydrates \\
\hline \multirow{2}{*}{ L-malic acid } & $\begin{array}{c}\text { Ammonium } \\
\text { sulfate }\end{array}$ & $\begin{array}{c}\text { Ammonium } \\
\text { acetate }\end{array}$ & Glucose \\
\cline { 3 - 4 } & & Sodium acetate & Fructose \\
\cline { 3 - 4 } & & & Sucrose \\
\hline
\end{tabular}

\subsection{Experimental}

\subsubsection{Synthesis of LTTMs}

Starting materials (HBD and HBA) with 1:1 molar ratio were mixed thoroughly in a three-neck round bottom flask with magnetic stirring to promote contact between the solid crystals. $1 \mathrm{ml}$ of distilled water was added into the mixtures for obtaining higher efficiency in the synthesis of LTTMs without hydrated constituents. The flask was heated up slowly by conventional oil bath heating. The heating process was stopped when all the solid particles was no longer visible. The reaction temperature and time taken to synthesize the LTTMs were recorded. The experiment was repeated with 1:2 and 1:3 molar ratio of malic acid to HBA.

\subsubsection{Physical properties of LTTMs}

The synthesized LTTMs were subjected to room temperature in order to ensure no crystallization of starting materials. The viscosity of the LTTMs was also observed while cooling the LTTMs from reaction temperature to room temperature.

\subsubsection{Fourier transform infrared spectroscopy (FTIR) analysis of LTTMs}

FTIR spectra of the starting materials and the LTTMs were analyzed using Frontier FT-IR Spectrometer (PerkinElmer). FTIR spectrometer simultaneously collects spectral data in a wide spectral range which enabled a significant advantage in measuring intensity over a narrow range of wavelengths at a time. The data collected was compared in order to ensure the existence of hydrogen bond between the starting materials. 


\section{Results and discussions}

\subsection{Compatibility of hydrogen bond acceptor with malic acid}

LTTMs consist of at least one hydrogen bond donor (HBD) and one hydrogen bond acceptor (HBA) counterpart that result in the formation of liquid mixture showing an unusually low freezing point. The building principles are not easy to generalize. Hydrogen bonds present different contact distances and binding energies which do not depend only on the donor and acceptor nature [11]. They often possess extremely low volatility and their properties can be adjusted by selecting the nature and ratio of hydrogen bonding pairs. The selection of the starting materials was made on the basis of the available functional groups and the key interactions involved in lignocellulosic biomass dissolution [9]. In general, LTTMs with malic acid as HBD were found to show higher efficiency in delignification when comparing with other hydrogen bond donors [9]. The synthesized LTTMs with carbohydrates as HBA in different molar ratio are shown in Figure 1. The LTTMs were formed in the temperature range of 60 to $100^{\circ} \mathrm{C}$ with reaction time of $10 \mathrm{~min}$.
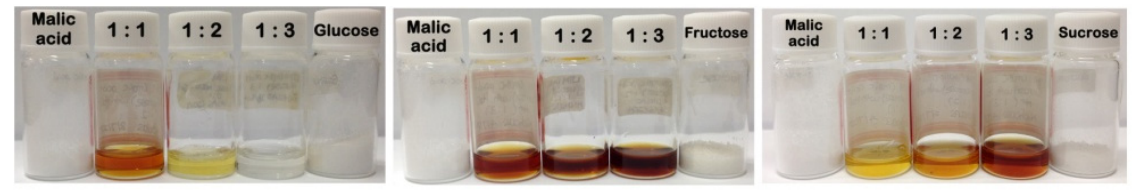

Figure 1: Synthesized LTTMs with carbohydrates as HBA.

On the other hand, organic salt (ammonium acetate) was not compatible with the malic acid as the mixtures still remained in solid state at temperature of $200^{\circ} \mathrm{C}$ for $30 \mathrm{~min}$. Figure 2 shows the LTTMs derived from ammonium sulfate and ammonium acetate as HBA. The LTTMs were formed at a higher reaction temperature range of 100 to $180^{\circ} \mathrm{C}$ with reaction time of $20 \mathrm{~min}$.

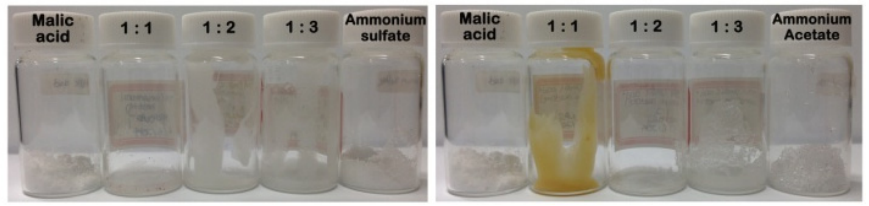

Figure 2: Synthesized LTTMs with ammonium sulfate and ammonium acetate as HBA.

The LTTMs prepared from carbohydrates remained in liquid state when exposed to room temperature while LTTMs derived from ammonium sulfate and ammonium acetate were crystallized. In this context, carbohydrates as HBA were more compatible with malic acid compared to the aforementioned organic and inorganic salt. The hydrogen bonding and van der Waals interactions prevented 
crystallization of the starting materials thus resulting in the mixtures remaining in liquid state [12]. Nevertheless, further investigation such as FTIR analysis was conducted in order to prove the existence of hydrogen bond in the starting materials which prevented the LTTMs from crystallization. The compatibilities of HBA with malic acid in the synthesis of LTTMs were tabulated in Table 2.

Table 2: Compatibility of HBA with malic acid.

\begin{tabular}{|c|c|c|c|}
\hline $\begin{array}{c}\text { Hydrogen bond } \\
\text { donor (HBD) }\end{array}$ & $\begin{array}{c}\text { Hydrogen bond } \\
\text { acceptor (HBA) }\end{array}$ & $\begin{array}{c}\text { Molar ratio } \\
\text { (HBD:HBA) }\end{array}$ & $\begin{array}{c}\text { Compatibility } \\
\text { (crystallization) }\end{array}$ \\
\hline \multirow{3}{*}{ L-malic acid } & Ammonium sulfate & $1: 1,1: 2,1: 3$ & Incompatible \\
\cline { 2 - 4 } & Ammonium acetate & $1: 1,1: 2,1: 3$ & Incompatible \\
\cline { 2 - 4 } & Sodium acetate & $1: 1,1: 2,1: 3$ & Incompatible \\
\cline { 2 - 4 } & Carbohydrates & $1: 1,1: 2,1: 3$ & Compatible \\
\hline
\end{tabular}

Besides, addition of small amount of distilled water in synthesis was necessary. The distilled water assisted in reducing the reaction temperature and time for the synthesis of LTTMs without hydrated constituents. It further helped to reduce the viscosity of the synthesized LTTMs. The dominance of water as solvent was due to its ability to form hydrogen bonds. Water is an ample natural resource which can act both as a donor and an acceptor. The water is assumed to form part of the solvent itself through stronger intermolecular bonds without affecting the characteristics of mixtures [12].

\subsection{Existence of hydrogen bond in LTTMs}

The acidity of the proton is responsible for the formation of an LTTM instead of ionic liquid (IL). A stronger base with a higher $\mathrm{pKa}$ needs to be facing the $\mathrm{H}$-bond donor or a stronger acid needs to be facing the acceptor for the formation of IL. The existence of hydrogen bonding can be verified as well by the shifts in the representative peaks of the involved bonds in the FTIR spectra [11].

FTIR analysis was carried out on the LTTMs derived from sucrose in order to prove the hydrogen bond restrained the solidification of initiating materials. The LTTMs synthesized from sucrose were chosen for the analysis as it is cheap and commercially available compared to glucose and fructose. The FTIR spectra for malic acid, sucrose and LTTMs of malic acid and sucrose (MS 1:1, MS 1:2, MS 1:3) are shown in Figure 3.

Figure 3 shows the representative peak of carboxylic acid group of malic acid with wavenumber $1710 \mathrm{~cm}^{-1}$ for the $\mathrm{C}=\mathrm{O}$ group turned into broader in the MS 1:1, MS 1:2 and MS 1:3 due to the hydrogen bonding. The peaks involved in the $\mathrm{H}-$ bonding responsible for the formation of the LTTM were shifted and broader within the wavenumber range of 2500 to $3600 \mathrm{~cm}^{-1}$ for the $\mathrm{OH}$ groups for carboxylic acid and alcohol). The main bands for the peak shifts and malic acid peaks were demarcated by dotted red lines. The results were comparable with the works of Francisco et al. [9] in which the hydrogen bonding existed between malic acid and proline as observed by FTIR analyses. 


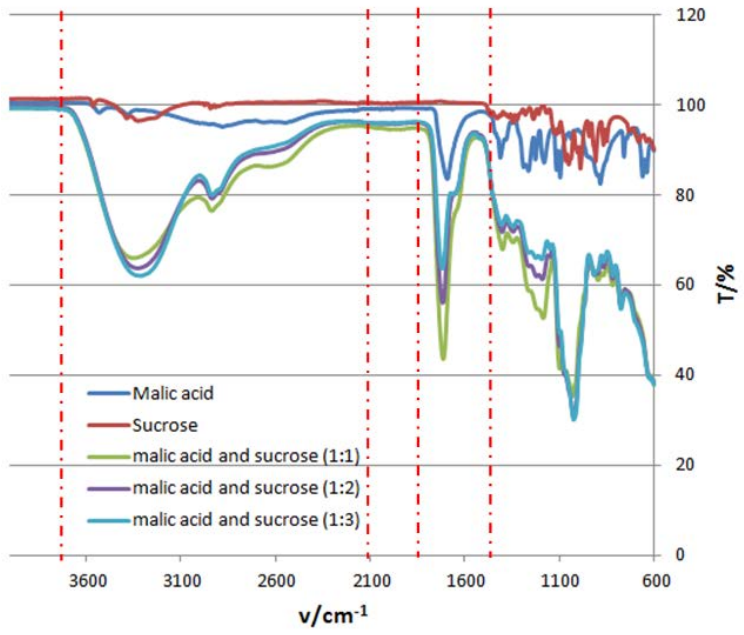

Figure 3: FTIR spectra of malic acid, sucrose and LTTMs of malic acid and sucrose (MS 1:1, MS 1:2, MS 1:3).

The LTTMs with three different molar ratio of malic acid to sucrose revealed the spectra which were in vicinity to each other. Hence, further study such as biopolymer solubility is crucial in studying the effect of increased molar ratio of sucrose. This screening enables the evaluation of the potential ability of LTTMs in delignifying the lignocellulosic biomass. In this context, LTTMs with high selectivity is prudent for isolating lignin from cellulose and hemicellulose.

\section{Conclusions}

In conclusion, carbohydrates such as glucose, fructose and sucrose were able to take the role of hydrogen bond acceptor (HBA) in the synthesis of LTTMs with malic acid as hydrogen bond donor (HBD). On the other hand, ammonium acetate, sodium acetate and ammonium sulfate were incompatible with malic acid due to the crystallization of starting materials when the mixtures were cooled down to room temperature. FTIR analysis of the LTTMs synthesized from sucrose proved the existence of hydrogen bond which helped keep the mixtures in liquid state. Further analysis such as biopolymer solubility is needed to distinguish the efficiency of delignification with increased molar ratio of sucrose in the synthesis of LTTMs.

\section{Acknowledgements}

The authors would like to acknowledge Kumamoto University and Universiti Teknologi PETRONAS for the support given to undertake the research work. The authors gratefully acknowledge the funding support from JSPS Bilateral Joint Research Program and Fundamental Research Grant Scheme (FRGS). 


\section{References}

[1] Nilsen, M.H., Antonakou, E., Bouzga, A., Lappas, A., Mathisen, K. \& Stocker, M., "Investigation of the effect of metal sites in the Me-AlMCM-41 ( $\mathrm{Me}=\mathrm{Fe}, \mathrm{Cu}$ or $\mathrm{Zn}$ ) on the catalytic behavior during the pyrolysis of wooden based biomass," Microporous and Mesoporous Materials, vol. 105, pp. 189-203, 2007.

[2] Hamzeh, Y., Ashori, A., Khorasani, Z., Abdulkani, A. \& Abyaz, A., "Preextraction of hemicelluloses from bagasse fibers: Effects of dry-strength additives on paper,” Industrial Crops and Products, vol. 43(5), pp. 365-371, 2013.

[3] Öhgren, K., Bura, R., Saddler, J. \& Zacchi, G., "Effect of hemicellulose and lignin removal on enzymatic hydrolysis of steam pretreated corn stover," Bioresource Technology, vol. 98(13), pp. 2503-2510, 2007.

[4] Yang, D., Zhong, L.X., Yuan, T.Q., Peng, X.W. \& Sun, R.C., “Studies on the structural characterization of lignin, hemicelluloses and cellulose fractionated by ionic liquid followed by alkaline extraction from bamboo," Industrial Crops and Products, vol. 43(1), pp. 141-149, 2013.

[5] Remsing, R.C., Swatloski, R.P., Rogers, R.D. \& Moyna, G., Mechanism of cellulose dissolution in the ionic liquid 1-n-butyl-3-methylimidazolium chloride: A 13C and 35/37Cl NMR relaxation study on model systems, Chem. Commun., vol. 12, pp. 1271-1273, 2006.

[6] Spronsen, J.V., Tavares, C., Witkamp, G., Jong, W. \& Kroon, M.C., Separation and recovery of the constituents from lignocellulosic biomass by using ionic liquids and acetic acid as co-solvents for mild hydrolysis, Chem. Eng. Process, vol. 50, pp. 196-199, 2011.

[7] Sun, N., Rodriguez, H., Rahman, M. \& Rogers, R.D., Where are Ionic Liquid Strategies Most Suited in the Pursuit of Chemicals and Energy from Lignocellulosic Biomass? Chem. Commun., vol. 47, pp. 1405-1421, 2011.

[8] Abbott, A.P., Boothby, D., Capper, G., Davies, D.L., Rasheed, R.K. \& Am J., Ionic Liquids in Biotransformations and Organocatalysis: Solvents and Beyond, Chem. Soc., vol. 126, pp. 9142-9147, 2004.

[9] Francisco, M., Bruinhorst, A. \& Kroon, M.C., New natural and renewable low transition temperature mixtures (LTTMs): screening as solvents for lignocellulosic biomass processing, Green Chem., vol. 14(8), pp. 2153-2157, 2012.

[10] Gilli, P., Pretto, L., Bertolasi V. \& Gilli, G., Predicting hydrogen-bond strengths from acid-base molecular properties. The $\mathrm{pK}(\mathrm{a})$ slide rule: toward the solution of a long-lasting problem, Acc. Chem. Res., vol. 42, pp. 33-44, 2009.

[11] Desiraju, G.R. \& Steiner, T., The weak hydrogen bond in structural chemistry and biology, Oxford University Press Inc., New York, 1999.

[12] Francisco, M., Bruinhorst, A. \& Kroon, M.C., low-transition-temperature mixtures (LTTMs): a new generation of designer solvent, Angew. Chem. Int. Ed., vol. 52, pp. 3074-3085, 2013. 